\title{
Synergistic Activation of Defense Responses in Arabidopsis by Simultaneous Loss of the GSL5 Callose Synthase and the EDR1 Protein Kinase
}

\author{
Anna Wawrzynska, Natalie L. Rodibaugh, and Roger W. Innes \\ Indiana University, Bloomington, IN 47405, U.S.A. \\ Submitted 23 September 2009. Accepted 19 January 2010.
}

\begin{abstract}
Loss-of-function mutations in the EDR1 gene of Arabidopsis confer enhanced resistance to Golovinomyces cichoracearum (powdery mildew). Disease resistance mediated by the edr1 mutation is dependent on an intact salicylic acid (SA) signaling pathway, but edr1 mutant plants do not constitutively express the SA-inducible gene $P R-1$ and are not dwarfed. To identify other components of the $E D R 1$ signaling network, we screened for mutations that enhanced the edr1 mutant phenotype. Here, we describe an enhancer of edr1 mutant, eed3, which forms spontaneous lesions in the absence of pathogen infection, constitutively expresses both SA- and methyl jasmonate (JA)-inducible defense genes, and is dwarfed. Positional cloning of eed 3 revealed that the mutation causes a premature stop codon in GLUCAN SYNTHASE-LIKE 5 (GSL5, also known as POWDERY MILDEW RESISTANT 4), which encodes a callose synthase required for pathogen-induced callose production. Significantly, gsl5 single mutants do not constitutively express $P R-1$ or AtERF1 (a JA-inducible gene) and are not dwarfed. Thus, loss of both EDR1 and GSL5 function has a synergistic effect. Our data suggest that EDR1 and GSL5 negatively regulate $\mathrm{SA}$ and $\mathrm{JA}$ production or signaling by independent mechanisms and that negative regulation of defense signaling by GSL5 may be independent of callose production.
\end{abstract}

To identify genes that regulate plant defense responses, we previously screened for Arabidopsis thaliana mutants with increased resistance to virulent pathogens. A mutant that displayed enhanced disease resistance (edrl) to Pseudomonas syringae and Golovinomyces cichoracearum (powdery mildew) was found (Frye and Innes 1998). The edrl-1 mutation causes a premature stop codon in the EDRl gene, which encodes a protein kinase with similarity to CTR1 (Frye et al. 2001), a negative regulator of ethylene responses (Cao et al. 1997; Kieber et al. 1993). The enhanced resistance of the $e d r l$ mutant is suppressed by mutations that block salicylic acid (SA) perception (nprl/nim1) or reduce SA production (sid2, eds1 and pad4) (Frye et al. 2001; Tang et al. 2005). The transgenic expression of $N a h G$, which lowers endogenous SA levels, also eliminates

Current address for A. Wawrzynska: Institute of Biochemistry and Biophysics, Polish Academy of Sciences, Pawin' skiego 5A Str, 02-106 Warsaw, Poland

Corresponding author: Roger W. Innes; E-mail: rinnes@indiana.edu; Fax: +1.812 .855 .6082 . the edrl-mediated enhanced disease resistance phenotype (Frye et al. 2001). In contrast to the clear requirement for SA signaling in $e d r l$-mediated resistance, neither ethylene nor jasmonic acid appear to be necessary, as mutations in the ETHYLENE INSENSITIVE 2 (EIN2) gene or CORONATINE INSENSITIVE 1 (COIl) gene do not alter edrl-mediated disease resistance (Frye et al. 2001).

SA is a central regulator of plant defense responses (Delaney et al. 1994). Exogenous application of SA induces a heightened state of resistance to numerous pathogens (Vernooij et al. 1994), while reduction of endogenous SA levels makes plants more susceptible to both virulent and avirulent pathogens (Delaney et al. 1994; Nawrath and Metraux 1999; Wildermuth et al. 2001). SA levels are elevated during pathogen infection and appear to enhance resistance, in part, by potentiating production of reactive oxygen species (Shirasu et al. 1997) along with inducing a large suite of defense genes (Glazebrook et al. 2003; Tsuda et al. 2008). Understanding how SA levels are regulated and how SA regulates defense responses is a major goal of the plant-microbe interaction field. We wish to understand the link between EDR1 function and activation of SAmediated defenses.

Although the EDR1 protein appears to function as a negative regulator of SA-mediated defenses, the $e d r l$ mutant does not contain significantly elevated SA levels and it does not constitutively express the $P R-1$ gene (Frye et al. 2001), which is often used as an indicator of SA signaling. Instead, the $e d r l$ mutant behaves as if it was primed for defense gene induction, similar to plants treated with $\beta$-aminobutyric acid (BABA) (van Hulten et al. 2006). This primed state leads to a more rapid and more robust defense response upon pathogen infection (Conrath et al. 2002; Frye and Innes 1998; Frye et al. 2001; Ton et al. 2005). BABA-induced priming is dependent, in part, on the hormone abscissic acid (ABA), as Arabidopsis mutants defective in ABA synthesis (abal-5) or ABA signaling (abi4-1) are impaired in BABA-induced resistance to the necrotrophic fungal pathogens Alternaria brassicicola and Plectosphaerella cucumerina (Ton and Mauch-Mani 2004). The role of ABA in this resistance appears to be linked to production of callose, a pathogen-induced $\beta$-1,3-glucan thought to function as a physical and chemical barrier to entry of fungal hyphae. BABA-treated plants show an earlier and more pronounced accumulation of callose upon pathogen infection, but callose accumulation is absent in BABA-treated abi4-1 plants. Furthermore, a mutation in the callose synthase gene GLUCAN SYNTHASE-LIKE GSL5 (also known as POWDERY MILDEW RESISTANT 4 [PMR4] but referred to as GSL5 in 
the remainder of this paper) blocks BABA-induced callose accumulation and resistance to Alternaria brassicicola and Plectosphaerella cucumerina (Ton and Mauch-Mani 2004).

The role of ABA signaling and callose synthesis in edrlmediated disease resistance is not yet clear. However, the $e d r l$ mutant displays enhanced sensitivity to ABA in a seedling germination assay (Wawrzynska et al. 2008). In addition, edrlmediated resistance can be suppressed by a gain-of-function mutation in the KEEP ON GOING (KEG) gene (Wawrzynska et al. 2008), which encodes a RING finger E3-ubiquitin ligase thought to target the transcription factor ABSCISIC ACIDINSENSITIVE5 (ABI5) for proteasome-mediated degradation (Stone et al. 2006). Loss-of-function keg mutant plants accumulate high levels of ABI5 and are hypersensitive to ABA (Stone et al. 2006). The gain-of-function keg4 mutation, which suppresses edrl-mediated resistance, also suppresses the hypersensitivity of $e d r l$ to ABA (Wawrzynska et al. 2008). Other phenotypes of the $e d r l$ mutant are enhanced drought-induced growth inhibition and enhanced ethylene-induced senescence (Frye et al. 2001; Tang et al. 2005), which are consistent with an overall enhanced sensitivity of edrl plants to ABA (Wawrzynska et al. 2008). There may also be a link to callose synthesis as, counterintuitively, loss of GSL5 function renders Arabidopsis resistant to powdery mildew infection via a mechanism that highly resembles $e d r l$-mediated resistance (Jacobs et al. 2003; Nishimura et al. 2003). Similar to infection of edrl plants, infection of pmr4-1 plants with G. cichoracearum induces large lesions that are not observed in wild-type (WT) plants and a more rapid induction of the defense gene $P R-1$ (Vogel and Somerville 2000). As observed with edrl, pmr4-1mediated resistance can be suppressed by mutations that reduce SA synthesis or SA perception (Nishimura et al. 2003). Despite these phenotypic similarities, the mechanistic links between callose synthesis, ABA signaling, SA signaling, and EDR1 function remain unknown.

To identify additional components of the EDR1 signaling network, we performed a mutant screen to identify both enhancers and suppressors of the edrl mutant phenotype (Wawrzynska et al. 2008). Such edrl suppressors and enhancers may uncover additional components of the SA regulatory system or pathways that interact with the EDR1 pathway in the regulation of defense responses as well as downstream signaling components. Here, we report the identification of an $e d r l$ enhancer mutant that shows severe growth retardation, necrotic lesions, and elevated $P R-1$ expression even in the absence of powdery mildew infection. Mapping of this enhancer mutation revealed that it was caused by a premature stop codon in the callose synthase gene GSL5, indicating that simultaneous loss of EDR 1 and GSL5 has a synergistic effect on the regulation of defense responses.

\section{RESULTS}

The eed 3 mutation causes retarded growth and necrotic lesion phenotypes that are dependent on the edr1 mutation.

We screened a population of Arabidopsis mutants (60,000 M2 seedlings derived from approximately 3,500 ethyl methane sulfonate-mutagenized M1 edrl plants) to identify mutations that suppressed or enhanced the edrl disease resistance phenotype (Wawrzynska et al. 2008). We identified nine edrl enhancers, i.e., plants displaying necrotic lesions prior to powdery mildew infection. The mutant with the severest phenotype was selected for further characterization and was named eed3 for enhancer of edrl mutant 3. In comparison to WT Col-0 and edrl plants, edrleed 3 double mutant plants were dwarfed and displayed large necrotic lesions, mainly on the older leaves starting to appear after 4 to 5 weeks of growth under short-day $(9$ h) conditions (Fig. 1). Crosses with WT Col-0 and edrl plants revealed that the edrleed 3 mutant phenotype required both mutations. We therefore proceeded with isolation of the EED3 gene.

\section{Identification of the $E E D 3$ gene.}

To map the eed 3 mutation, we crossed the edrleed 3 mutant to Landsberg erecta (Ler) and, then, selected F2 plants that displayed a normal edrl mutant phenotype (i.e., WT appearance prior to inoculation with powdery mildew and development of lesions after inoculation). These plants were confirmed to be homozygous for $e d r l$ using polymerase chain reaction (PCR) and were then allowed to self. The resulting F3 families were scored for segregation of the dwarfed phenotype. A total of 218 F3 plants (derived from selfing 37 F2 parents homozygous for $e d r l$ ) displaying the dwarf phenotype were selected for DNA isolation and scoring of molecular markers (described below).

Initially, the eed3 mutation was mapped to a region between the microsatellite markers CIW5 and T7M24 on chromosome 4
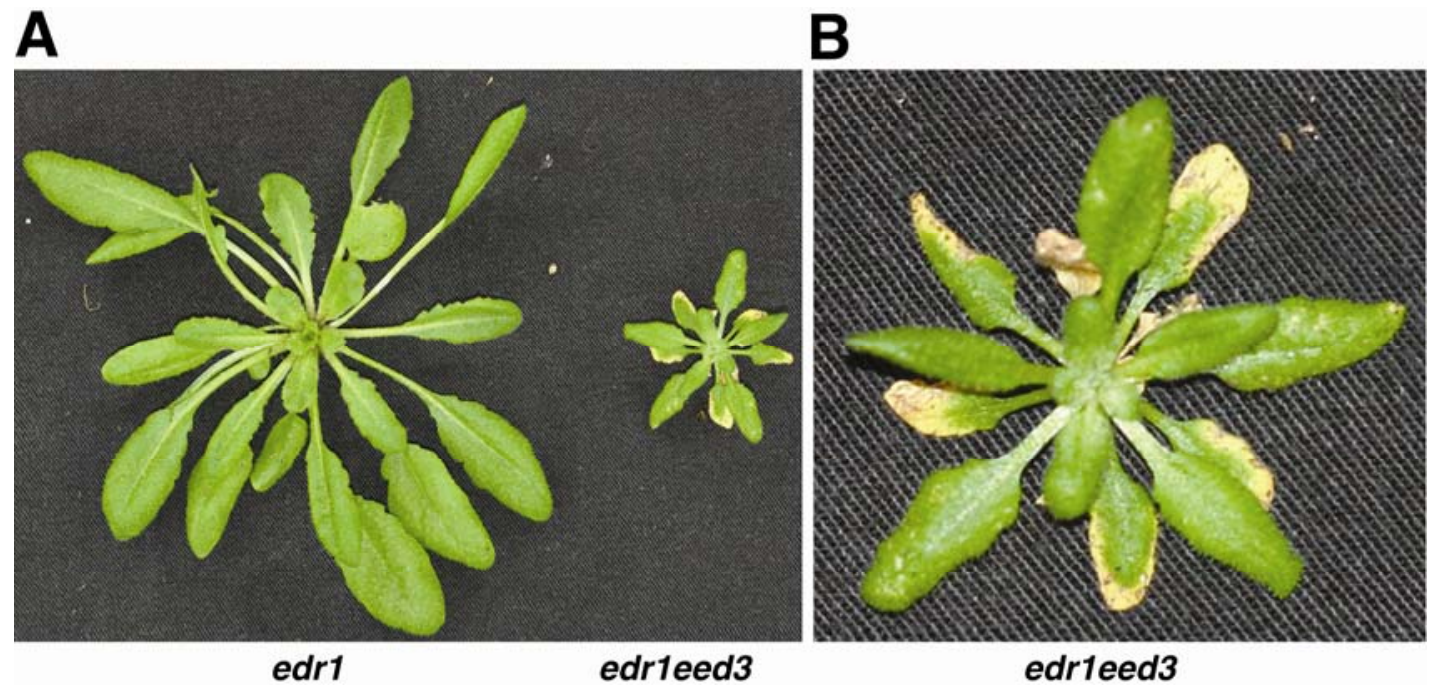

Fig. 1. Phenotype of the edrleed 3 mutant. A, edrl and edrleed 3 plants at 5 weeks of growth under short days. B, Close-up of the edrleed 3 mutant. Note the large necroses on the edges of older leaves and small necroses on the younger leaves. 
(Fig. 2). To further localize the mutated gene, we created PCR markers at intervals between these two markers using small insertions or deletions that are polymorphic between Ler and Col-0 (Jander et al. 2002). Scoring of these additional markers localized the mutation to a $160-\mathrm{kb}$ interval (covering the $3^{\prime}$ end of bacterial artificial chromosome [BAC] F9H3 [GenBank accession AF071527] and the 5' end of BAC T5L23 [GenBank accession AC005142]) defined by one recombinant at the left border and 12 recombinants at the right border (Fig. 2). This region harbors 47 genes (from At4g03390 to At4g03730). Among them, we found the GSL5 gene (At4g03550) encoding callose synthase. Loss-of-function mutants of GLS5 are known to have enhanced resistance to powdery mildew (Jacobs et al. 2003; Nishimura et al. 2003). Therefore, we amplified GSL5 from the edrleed 3 mutant and sequenced it. Sequencing revealed a $G$ to $A$ transition in the third exon, which caused a premature stop codon at amino acid 1,559 of this 1,780-amino acid protein. Because the premature stop codon is 24 amino acids upstream of an RXTG motif believed to be involved in UDP-glucose binding in this enzyme family (Inoue et al. 1996; Ostergaard et al. 2002) and eliminates the last five transmembrane domains, it is highly unlikely that the truncated protein would have any callose synthase activity.

\section{Phenotype of edr1 pmr4-1 double mutant.}

To confirm that loss of GSL5 function enhances the edr1 phenotype, we crossed $e d r l$ plants to the previously described pmr4-1 mutant, which contains a stop codon at amino acid 687, near the beginning of the second exon (Fig. 2) (Nishimura et al. 2003; Vogel and Somerville 2000). In the F2 generation, we observed dwarfed plants with lesions segregating at a ratio of 4:70, which is not significantly different from the expected ratio of 1:15 (chi squared $=0.09, P=0.76$ ). Genotyping of three dwarfed plants revealed that all were homozygous for both $e d r l$ and pmr4-1, while genotyping of three sibling plants with
WT stature showed that none were homozygous for both mutations. Thus, we conclude that the loss of GSL5 function does, indeed, dramatically enhance the edrl phenotype and that pmr4-1 and edrl mutations have a synergistic effect.

\section{Powdery mildew growth is inhibited in edrleed 3 plants.}

To further characterize edrleed 3 double mutants, we looked at the development of $G$. cichoracearum and host cell death using trypan-blue staining. We also examined callose deposition during powdery mildew infection with aniline-blue staining. Spores of G. cichoracearum germinated and produced appressorial germ tubes on WT Col-0, edrl, and edrleed3 leaves 1 day after inoculation (data not shown). By 3 days after inoculation, G. cichoracearum developed extensive branched hyphae on both Col-0 and edrl leaves, while fungal growth was significantly inhibited on leaves of edrleed 3 (data not shown). By 5 days, extensive hyphae nearly covered both Col-0 and edrl leaves but not edrleed3 leaves (Fig. 3A). Many conidiophores formed on Col-0 leaves, while significantly fewer formed on edrl leaves, consistent with our previous analyses of the edrl mutant (Frye and Innes 1998). These observations demonstrated that the growth of G. cichoracearum was affected at a late stage of the infection process in edrl mutant but at an earlier stage in the edrleed 3 mutant. The edrl mutant displayed large patches of dead mesophyll cells 5 days after infection (Fig. 3B), while edrleed 3 plants displayed large necroses even before infection. This massive mesophyll cell death prior to infection was not observed on WT Col-0 leaves nor in pmr4-1 mutant leaves (Nishimura et al. 2003; Vogel and Somerville 2000).

Both WT Col-0 and edrl plants displayed punctate staining of callose in the cell walls of epidermal cells 5 days after inoculation (Fig. 3C); however, only edrl showed callose staining in large clumps of mesophyll cells (Fig.3). This is consistent with our previous results (Frye and Innes 1998). As expected, no

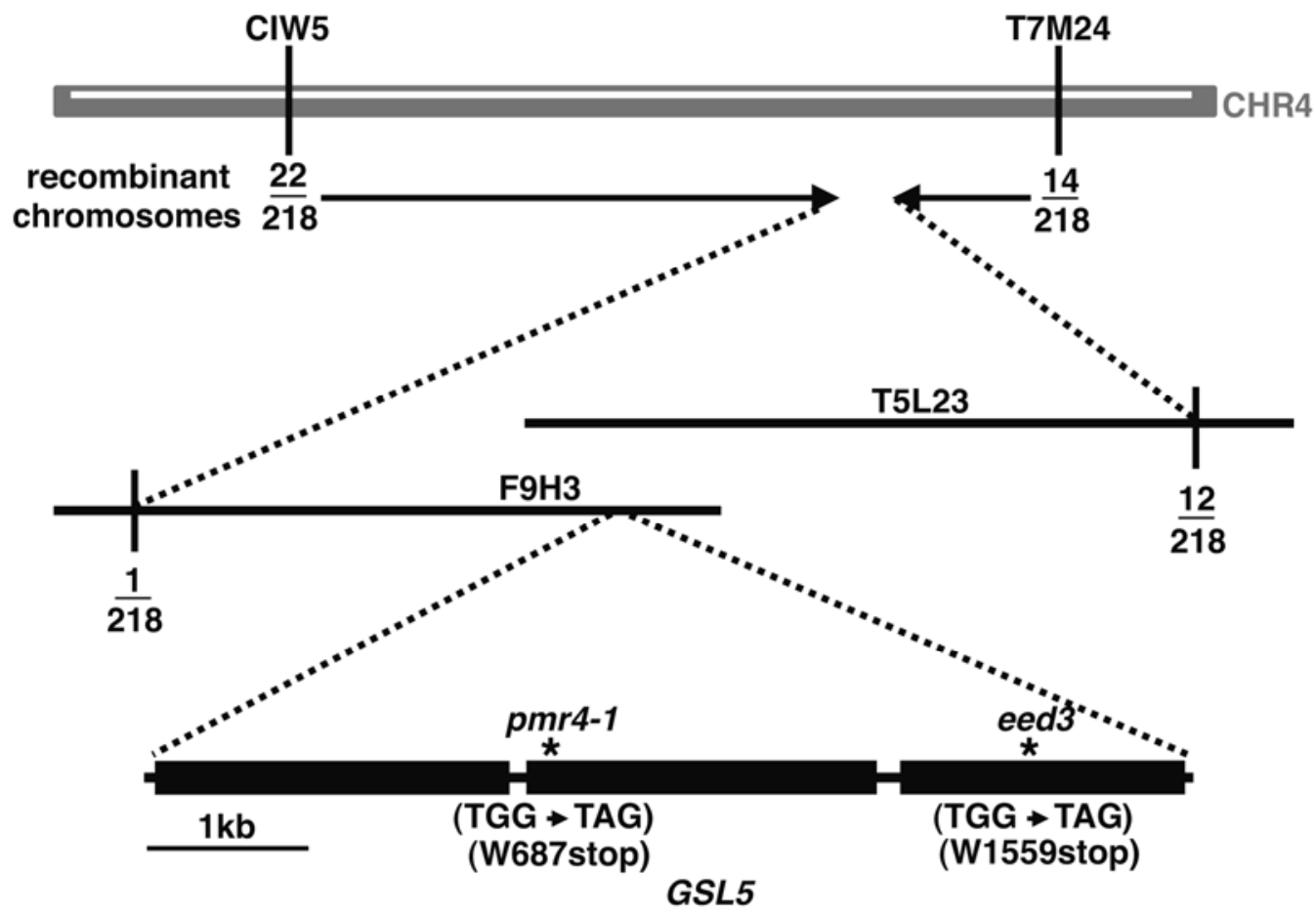

Fig. 2. Positional cloning of EED3. Vertical lines indicate the positions of markers that defined the eed3 genetic interval. Fractions indicate the number of recombination events identified between the indicated markers and the EED3 gene out of the total number of chromosomes scored. Bacterial artificial chromosome clones spanning the region to which eed3 was mapped are indicated. Sequencing of candidate genes in this interval revealed a $\mathrm{G}$ to A transition in At4g03550 (GSL5), resulting in a premature stop codon at tryptophan 1,559. Also shown is the genomic structure of the GSL5 gene, with exons indicated by black boxes and positions of the pmr4-1 and eed3 mutations indicated by the * symbol. 
callose deposition was observed in the leaves of edrleed 3 (Fig. 3C).

\section{GSL5 negatively regulates defense gene expression.}

Because the pmr4-1 mutant displays a more rapid induction of SA-inducible genes upon pathogen attack (Vogel and Somerville 2000) and because mutants with constitutively elevated SA levels typically have retarded growth (Andreasson et al. 2005; Jirage et al. 2001; Mateo et al. 2006; Mauch et al. 2001), we decided to analyze the edrleed 3 double mutant for expression of defense genes, to see if these were constitutively elevated. Specifically, we performed quantitative reverse transcriptase (qRT)-PCR analysis on $P R-1$ (At2g14610, a marker for SA-dependent gene expression [Bowling et al. 1994]), PBS3 (At5g13320, involved in regulation of SA levels [Nobuta et al. 2007]), ICS1 (At1g74710, involved in SA biosynthesis [Wildermuth et al. 2001]), AtERF1 (At4g17500, an APETALA2/ETHYLENE RESPONSE FACTOR transcription factor family member that is strongly inducible by methyl jasmonate [JA] [Nemhauser et al. 2006] and is upregulated in the edrl mutant [Wawrzynska et al. 2008]), SRG1-like (At4g10500, a stress-induced ascorbate oxidase upregulated in pmr4-1 after powdery mildew infection [Nishimura et al. 2003]), and an endochitinase (At2g43570, also upregulated in pmr4-1 following powdery mildew infection [Nishimura et al. 2003] and by the SA-responsive transcription factor WRKY70
[Li et al. 2004]). RNA was isolated from rosette leaves of 4.5week-old plants grown in the absence of pathogens under 9-h days, which was approximately 1 week after lesions began appearing on edrleed3 plants. All six of these defense genes were dramatically elevated in edrleed 3 plants compared with WT Col-0, edrl, and pmr4-1 plants (Fig. 4). This result suggests that callose or callose synthase acts to suppress both SAand JA-induced defense signaling in edrl mutant plants and that loss of both GSL5 and EDRI has a synergistic effect on both the SA and JA signaling pathways.

\section{DISCUSSION}

Arabidopsis contains a family of 12 putative callose synthase genes (GSL1 through GSL12) (Enns et al. 2005). Very limited information is available regarding the biological functions of individual GSL family members and the role of callose in plant development. Presumably, each member of the family mediates the synthesis of callose either in different tissues or under different environmental and developmental conditions or both (Richmond and Somerville 2000; Verma and Hong 2001). GSL5 appears to be the main biosynthetic enzyme responsible for callose deposition in response to biotic, abiotic, and chemical stresses (Nishimura et al. 2003). However, GSL5 transcript can be detected in all plant organs, including root, rosette leaf, stems, and flowers, and is apparently partially
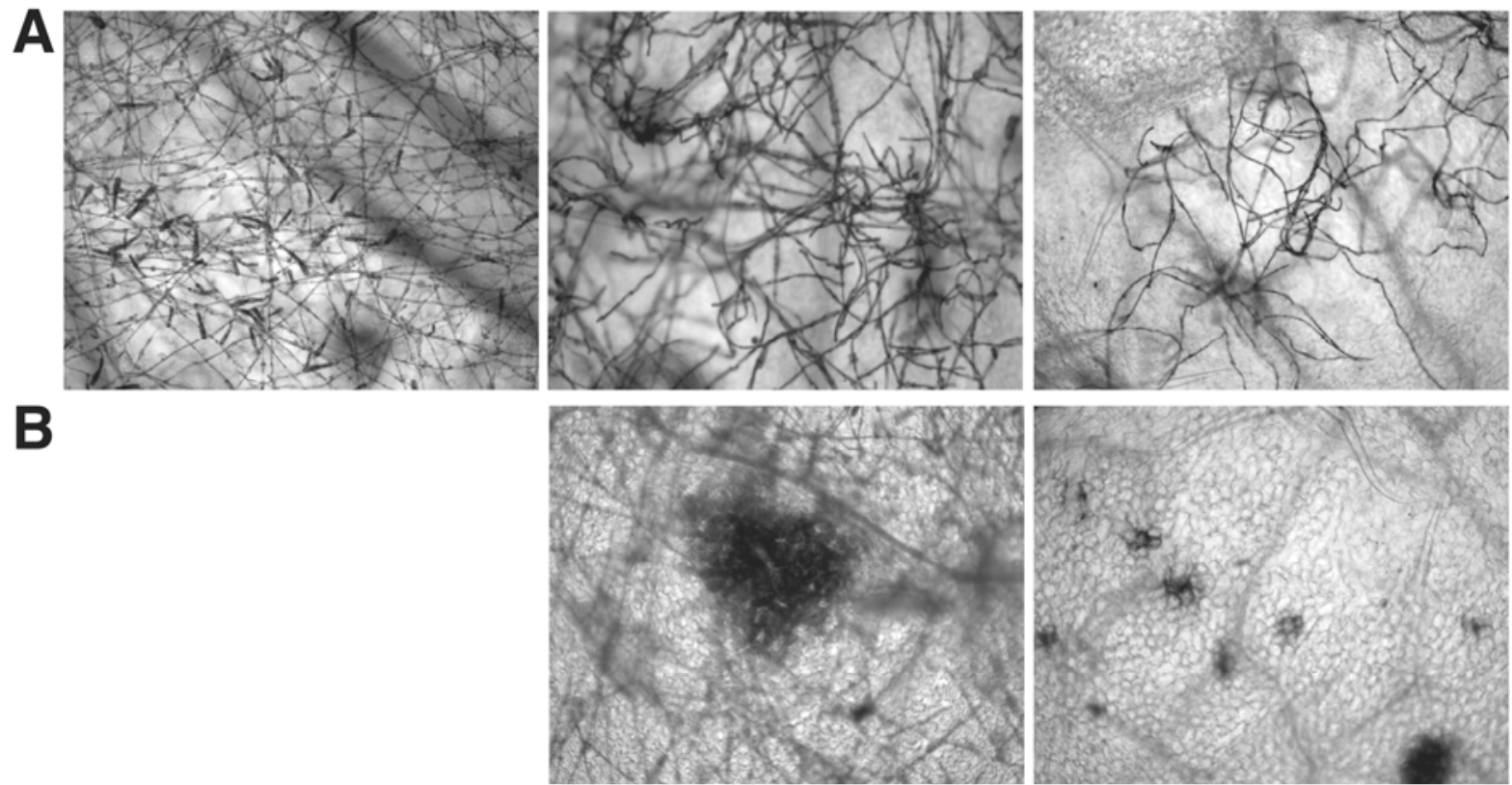

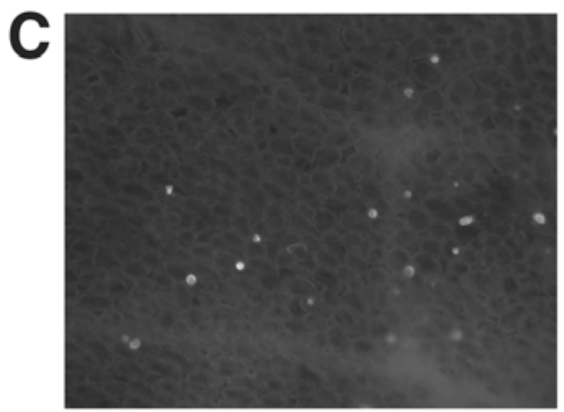

Col-0

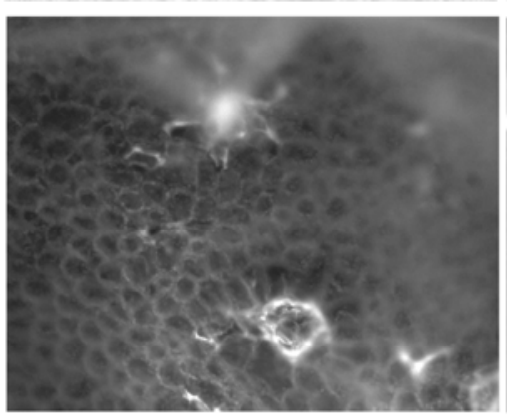

edr1

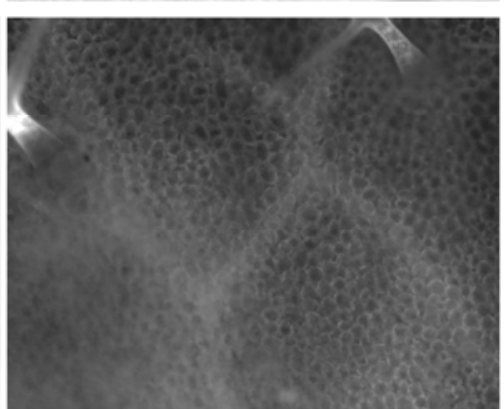

edr1eed3

Fig. 3. Response of Arabidopsis wild-type (WT) Col-0, edr1, and edr1eed3 plants to Golovinomyces cichoracearum. A, Fungal hyphae growing on the surface of WT Col-0, edrl, and edrleed 3 leaves 5 days after infection and stained with trypan blue. Note extensive hyphae and conidia produced on WT Col0 leaves 5 days after infection but very few conidia on $e d r 1$ leaves and limited hyphae growth on edrleed 3 leaves. B, Extensive mesophyll cell death in $e d r 1$ and edrleed 3 mutant leaves 5 days after infection revealed by trypan-blue staining $\mathbf{C}$, Aniline-blue staining for callose deposition in mesophyll cell walls of WT Col-0, edrl, and edrleed 3 leaves 5 days after inoculation. 
redundant in function with GSL1 during normal plant development (Enns et al. 2005). Plants heterozygous for a gsll mutation and homozygous for a gsl5 mutation are dwarfed and nearly infertile. The infertility is caused, in part, by nonviability of gsllgsl5 pollen, which lack a callose wall between the microspores of the pollen tetrad (Enns et al. 2005). Based on our experiments and those of Nishimura and associates (2003), we conclude that, during normal plant development, GSL5 also functions as a negative regulator of SA and JA biosynthesis or signaling or both.

A gsl5 single mutant (pmr4-1) grows at the same rate as WT and does not outwardly appear to be stressed, although, when grown under short-day conditions, leaves are epinastic (Vogel and Somerville 2000). Unlike previously identified classes of Arabidopsis disease-resistant mutants, $c p r, l s d$, and $a c d$ (Bowling et al. 1994; Dietrich et al. 1994), gsl5 plants do not constitutively express $P R-1$. However, when a $g s l 5$ mutation was combined with the $e d r l$ mutation, we observed a strong induction of the SA biosynthetic gene ICSI and the SA-inducible gene $P R-1$ (Fig. 4). Thus, we conclude that the EDR1 kinase must function in part to negatively regulate SA biosynthesis in the gsl5 mutant background. When infected with powdery mildew, gsl5 mutants develop lesions reminiscent of hypersensitive cell death (Vogel and Somerville 2000), similar to those observed for the edrl mutant (Frye and Innes 1998). Also like the $e d r l$ mutant, blocking the SA signaling pathway in a $g s l 5$ mutant suppresses the lesion phenotype and the enhanced resistance to powdery mildew (Nishimura et al. 2003). This indicates that the enhanced resistance of $g s l 5$ as well as of $e d r l$ is caused by a hyperactive SA response and is not a direct result of the loss of callose in $g s l 5$ mutants or kinase action in $e d r 1$ mutants. Combining gsl5 and $e d r l$ mutations has a synergistic effect on SA signaling, which suggests that GSL5 and EDR1 may negatively regulate SA signaling by separate pathways.

GSL5 mRNA was shown to accumulate in SA-treated Arabidopsis (Ostergaard et al. 2002) as well as in the Arabidopsis mpk4 mutant, which exhibits constitutive systemic acquired resistance and high levels of SA (Petersen et al. 2000). Nishimura and associates (2003) hypothesized that rapid callose deposition during the early stages of infection initiated by SA may then, in turn, repress later defense responses that may potentially be harmful for the plant. However, our data suggest that callose deposition may not be the primary mechanism of defense gene suppression by GSL5. We conclude this because, in uninfected edrl mutant plants, little to no callose is detect-

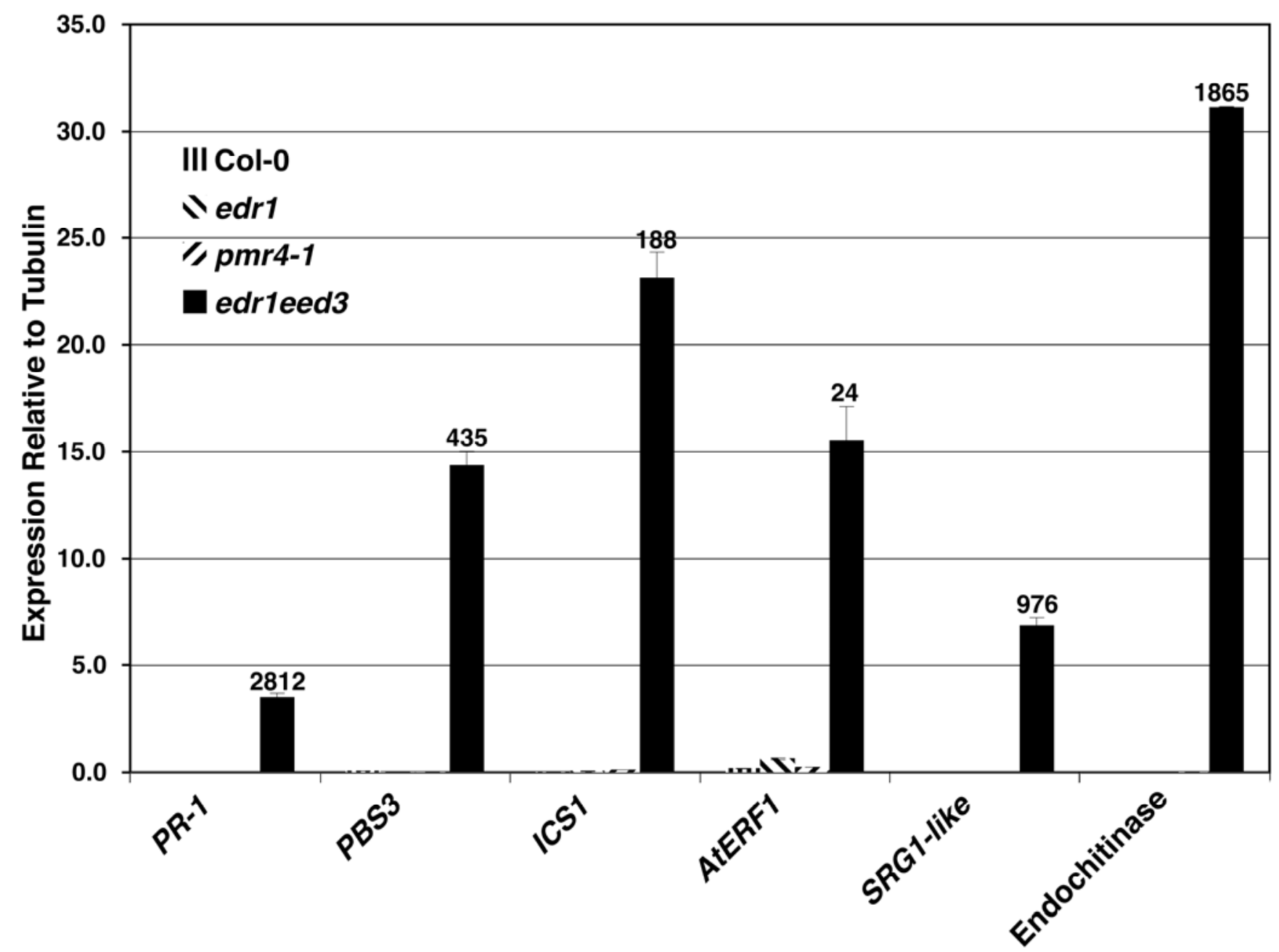

Fig. 4. Synergistic effect of $e d r 1$ and eed3 mutations on both salicylic acid- and jasmonate-inducible defense gene expression. Quantitative reverse transcriptase-polymerase chain reaction analysis was performed on the indicated defense genes in wild-type Col-0, edr1, pmr4-1, and edr1eed3 mutants grown for 4.5 weeks under 9-h days. Total RNA was isolated from leaves. Values are the average with standard deviation of three technical replicates and are normalized relative to the TUA3 alpha tubulin gene. Numbers above the edrleed 3 bar indicate the fold increase in expression relative to expression in the edrl or pmr4-1 single mutant (the mutant with the higher expression was used as the denominator). The whole experiment was repeated three times with similar results. Arabidopsis Genome Initiative locus numbers for the indicated genes are: PR-1, At2g14610; PBS3, At5g13320; ICS1, At1g74710; AtERF1, At4g17500; SRG1-like, At4g10500; endochitinase, At2g43570. 
able by aniline-blue staining and there is very low-level expression of $P R-1$, yet loss of GSL5 function in the $e d r 1$ mutant background leads to high-level expression of $P R-1$, even in the absence of pathogen infection (Fig. 4). Since there was no detectable callose in the $e d r l$ mutant leaves prior to infection, it seems unlikely that callose could have been responsible for suppressing $P R-1$ expression in these same leaves. If callose is not directly responsible for suppression of SA signaling in the edrl mutant, it would suggest that GSL5 has functions in addition to stress-induced callose biosynthesis. As mentioned above, GSL5 likely works in concert with GSL1 during normal plant development and likely contributes to normal cellular homeostasis (Enns et al. 2005). We speculate that it is this disruption in normal cellular homeostasis, when combined with loss of EDR1 function, that leads to massive induction of SA signaling.

Surprisingly, we also observed highly elevated expression of the JA-inducible gene AtERF1 in the edrleed 3 double mutant (Fig. 4). This is surprising because SA and JA signaling pathways often function antagonistically to each other (Kunkel and Brooks 2002) and the pmr4-1 mutant has been shown to produce less JA than WT plants during infection by Alternaria brassicicola (Flors et al. 2008). However, we have previously observed that $e d r l$ single mutant plants have modestly elevated expression of AtERFl as well as enhanced responsiveness to exogenous ABA (Wawrzynska et al. 2008), suggesting that EDR1 negatively regulates multiple hormone signaling pathways. We hypothesize that loss of GSL5 somehow induces SA and JA signaling that, in the absence of EDR1 function, reaches deleterious levels. In this context, it is noteworthy that overexpression of AtERF1 is known to cause dwarfing in Arabidopsis (Pre et al. 2008), as does constitutive elevation of SA signaling (Bowling et al. 1994); thus, the severely dwarfed stature of the edrleed 3 mutant may be a consequence of elevating both pathways.

\section{MATERIALS AND METHODS}

\section{Plant growth conditions.}

Arabidopsis thaliana plants were grown in growth rooms under a 9-h light and $15-\mathrm{h}$ dark cycle at $23^{\circ} \mathrm{C}$ as described previously (Frye and Innes 1998).

\section{Pathogen infections.}

G. cichoracearum UCSC1 was maintained on hypersusceptible A. thaliana pad4-2 mutant plants. Plants were inoculated between 4 and 6 weeks of age by gently brushing the leaves of diseased and healthy plants together to pass the conidia (asexual spores).

\section{Genetic and physical mapping of eed3.}

Genetic mapping was accomplished using an F2 population derived from a cross between the edrleed 3 mutant (Columbia genotype, Col-0) and Landsberg erecta (Ler). F2 seeds were planted and scored for disease resistance to G. cichoracearum as described above. Plants displaying an $e d r l$ phenotype were confirmed to be homozygous for the edrl mutation, using a PCR-based assay, and were then allowed to self to generate F3 families. Progeny from 37 F3 families were planted and scored for the eed3 phenotype (dwarfed plants with lesions). Genomic DNA was isolated from a total of 218 dwarfed F3 plants and was scored with published microsatellite markers. This initial mapping localized the eed 3 mutation between molecular markers CIW5 and T7M24 on chromosome 4. New molecular markers at intervals between these two markers were developed using the Monsanto Col-0 and Ler polymorphism database (primer sequences available upon request). These analyses localized the eed 3 mutation to a $160-\mathrm{kb}$ interval on chromosome 4, spanning Arabidopsis BAC clones F9H3 and T5L23.

\section{DNA sequencing.}

The GSL5 gene was amplified from the eed3 mutant by PCR and was directly sequenced. All sequencing reactions were performed using BigDye terminator kits (Applied Biosystems, Foster City, CA, U.S.A.) and were separated on an ABI 3730 automated DNA sequencer (Applied Biosystems).

\section{Analysis of fungal infection, callose deposition, and dead cells in Arabidopsis leaves.}

Fungal structures and dead plant cells were stained using alcoholic trypan blue (Koch and Slusarenko 1990). Callose deposition was detected by aniline-blue staining, as described by Adam and Somerville (1996). Stained leaves were mounted under coverslips with $50 \%$ glycerol and were observed with a Nikon e800 microscope.

\section{qRT-PCR analysis.}

Plants were grown under 9-h days, and RNA was isolated from leaves of WT Col-0, edrl, pmr4-1, and edrleed3 at 4.5 weeks. Total RNA was isolated using the Qiagen RNeasy kit and was treated with DNase (Invitrogen, Carlsbad, CA, U.S.A.) to remove DNA contamination. The High Capacity reverse transcriptase kit (Applied Biosystems) was utilized to obtain cDNA, and the samples were purified with Qiagen QIAquick PCR purification kit. qRT-PCR was performed using primers for $P R-1$ (At2g14610, 5'-CATGGGACCTACGCCTACC-3' and 5'-TTCTTCCCTCGAAAGCTCAA-3'), PBS3 (At5g13320, 5' ACTGGATTCTTGCTAAGTTCTG-3' and 5'-CACACCTTT CACATGCTTGGTT-3'), ICS1 (At1g74710, 5'-AAACACGC CTGAGAGACTATT-3' and 5'-TCTTTCGGACTGGTTAGT AAGT-3'), AtERF1 (At4g17500, 5'-GAGATTTGCCGTTGAA AGAA-3' and 5'-GTCGGAAGACGAAGAAGACG-3'), SRG1like (At4g10500, 5'-GACCAAATGCAGGTCATAAG-3' and 5'-GGGAAATAGAAAGTCGGAAT-3'), and endochitinase (At2g43570, 5'-AAGAAACAGGGTTCATGTGT-3' and 5'ACTCTGGTTTCTCTGTGTCG-3'). An alpha-tubulin gene, TUA3 (At5g19770, 5'-GTATTGAACGCATCGTGTG-3' and 5'-TGGGAGCTTTACTGTCTCGAA-3'), was used as a control for normalizing the amount of cDNA. Takara SYBR Premix Extaq was used for all qRT-PCR reactions and samples analyzed on an Mx3000P qPCR instrument (Stratagene, La Jolla, CA, U.S.A.).

\section{ACKNOWLEDGMENTS}

We thank the Arabidopsis Biological Resource Center at Ohio State for providing pmr4-1 mutant seed (ABRC stock number CS3858). This work was supported by a grant from the National Institute of General Medical Sciences of the National Institute of Health (grant number R01 GM063761 to R.W. I.).

\section{LITERATURE CITED}

Adam, L., and Somerville, S. C. 1996. Genetic characterization of five powdery mildew disease resistance loci in Arabidopsis thaliana. Plant J. 9:341-356.

Andreasson, E., Jenkins, T., Brodersen, P., Thorgrimsen, S., Petersen, N. H., Zhu, S., Qiu, J. L., Micheelsen, P., Rocher, A., Petersen, M., Newman, M. A., Bjorn Nielsen, H., Hirt, H., Somssich, I., Mattsson, O., and Mundy, J. 2005. The MAP kinase substrate MKS1 is a regulator of plant defense responses. Embo J. 24:2579-2589.

Bowling, S. A., Guo, A., Cao, H., Gordon, A. S., Klessig, D. F., and Dong, X. 1994. A mutation in Arabidopsis that leads to constitutive expression of systemic acquired resistance. Plant Cell 6:1845-1857.

Cao, H., Glazebrook, J., Clarke, J. D., Volko, S., and Dong, X. 1997. The Arabidopsis NPRl gene that controls systemic acquired resistance encodes a novel protein containing ankyrin repeats. Cell 88:57-63. 
Conrath, U., Pieterse, C. M., and Mauch-Mani, B. 2002. Priming in plantpathogen interactions. Trends Plant Sci. 7:210-216.

Delaney, T. P., Uknes, S., Vernooij, B., Friedrich, L., Weymann, K., Negrotto, D., Gaffney, T., Gut-Rella, M., Kessmann, H., Ward, E., and Ryals, J. 1994. A central role of salicylic acid in plant disease resistance. Science 266:1247-1250.

Dietrich, R. A., Delaney, T. P., Uknes, S. J., Ward, E. R., Ryals, J. A., and Dangl, J. L. 1994. Arabidopsis mutants simulating disease resistance response. Cell 77:565-577

Enns, L. C., Kanaoka, M. M., Torii, K. U., Comai, L., Okada, K., and Cleland, R. E. 2005. Two callose synthases, GSL1 and GSL5, play an essential and redundant role in plant and pollen development and in fertility. Plant Mol. Biol. 58:333-349.

Flors, V., Ton, J., van Doorn, R., Jakab, G., Garcia-Agustin, P., and Mauch-Mani, B. 2008. Interplay between JA, SA and ABA signalling during basal and induced resistance against Pseudomonas syringae and Alternaria brassicicola. Plant J. 54:81-92.

Frye, C. A., and Innes, R. W. 1998. An Arabidopsis mutant with enhanced resistance to powdery mildew. Plant Cell 10:947-956.

Frye, C. A., Tang, D., and Innes, R. W. 2001. Negative regulation of defense responses in plants by a conserved MAPKK kinase. Proc. Natl. Acad. Sci. U.S.A. 98:373-378.

Glazebrook, J., Chen, W., Estes, B., Chang, H. S., Nawrath, C., Metraux, J. P., Zhu, T., and Katagiri, F. 2003. Topology of the network integrating salicylate and jasmonate signal transduction derived from global expression phenotyping. Plant J. 34:217-228

Inoue, S. B., Qadota, H., Arisawa, M., Anraku, Y., Watanabe, T., and Ohya, Y. 1996. Signaling toward yeast 1,3-beta-glucan synthesis. Cell Struct. Funct. 21:395-402.

Jacobs, A. K., Lipka, V., Burton, R. A., Panstruga, R., Strizhov, N., SchulzeLefert, P., and Fincher, G. B. 2003. An Arabidopsis callose synthase, GSL5, is required for wound and papillary callose formation. Plant Cell 15:2503-2513.

Jander, G., Norris, S. R., Rounsley, S. D., Bush, D. F., Levin, I. M., and Last, R. L. 2002. Arabidopsis map-based cloning in the post-genome era. Plant Physiol. 129:440-450.

Jirage, D., Zhou, N., Cooper, B., Clarke, J. D., Dong, X., and Glazebrook, J. 2001. Constitutive salicylic acid-dependent signaling in cprl and cpr6 mutants requires PAD4. Plant J. 26:395-407.

Kieber, J. J., Rothenberg, M., Roman, G., Feldmann, K. A., and Ecker, J R. 1993. CTR1, a negative regulator of the ethylene response pathway in Arabidopsis, encodes a member of the raf family of protein kinases. Cell 72:427-441.

Koch, E., and Slusarenko, A. 1990. Arabidopsis is susceptible to infection by a downy mildew fungus. Plant Cell 2:437-445.

Kunkel, B. N., and Brooks, D. M. 2002. Cross talk between signaling pathways in pathogen defense. Curr. Opin. Plant Biol. 5:325-331.

Li, J., Brader, G., and Palva, E. T. 2004. The WRKY70 transcription factor: A node of convergence for jasmonate-mediated and salicylate-mediated signals in plant defense. Plant Cell 16:319-331.

Mateo, A., Funck, D., Muhlenbock, P., Kular, B., Mullineaux, P. M., and Karpinski, S. 2006. Controlled levels of salicylic acid are required for optimal photosynthesis and redox homeostasis. J. Exp. Bot. 57:17951807.

Mauch, F., Mauch-Mani, B., Gaille, C., Kull, B., Haas, D., and Reimmann, C. 2001. Manipulation of salicylate content in Arabidopsis thaliana by the expression of an engineered bacterial salicylate synthase. Plant J. 25:67-77.

Nawrath, C., and Metraux, J. P. 1999. Salicylic acid induction-deficient mutants of Arabidopsis express $P R-2$ and $P R-5$ and accumulate high levels of camalexin after pathogen inoculation. Plant Cell 11:1393-1404.

Nemhauser, J. L., Hong, F., and Chory, J. 2006. Different plant hormones regulate similar processes through largely nonoverlapping transcriptional responses. Cell 126:467-475.

Nishimura, M. T., Stein, M., Hou, B. H., Vogel, J. P., Edwards, H., and
Somerville, S. C. 2003. Loss of a callose synthase results in salicylic acid-dependent disease resistance. Science 301:969-972.

Nobuta, K., Okrent, R. A., Stoutemyer, M., Rodibaugh, N., Kempema, L., Wildermuth, M. C., and Innes, R. W. 2007. The GH3 acyl adenylase family member PBS3 regulates salicylic acid-dependent defense responses in Arabidopsis. Plant Physiol. 144:1144-1156.

Ostergaard, L., Petersen, M., Mattsson, O., and Mundy, J. 2002. An Arabidopsis callose synthase. Plant Mol. Biol. 49:559-566.

Petersen, M., Brodersen, P., Naested, H., Andreasson, E., Lindhart, U., Johansen, B., Nielsen, H. B., Lacy, M., Austin, M. J., Parker, J. E., Sharma, S. B., Klessig, D. F., Martienssen, R., Mattsson, O., Jensen, A. B., and Mundy, J. 2000. Arabidopsis map kinase 4 negatively regulates systemic acquired resistance. Cell 103:1111-1120.

Pre, M., Atallah, M., Champion, A., De Vos, M., Pieterse, C. M., and Memelink, J. 2008. The AP2/ERF-domain transcription factor ORA59 integrates jasmonic acid and ethylene signals in plant defense. Plant Physiol. 147:1347-1357.

Richmond, T. A., and Somerville, C. R. 2000. The cellulose synthase superfamily. Plant Physiol. 124:495-498.

Shirasu, K., Nakajima, H., Rajasekhar, V. K., Dixon, R. A., and Lamb, C. 1997. Salicylic acid potentiates an agonist-dependent gain control that amplifies pathogen signals in the activation of defense mechanisms. Plant Cell 9:261-270.

Stone, S. L., Williams, L. A., Farmer, L. M., Vierstra, R. D., and Callis, J. 2006. KEEP ON GOING, a RING E3 ligase essential for Arabidopsis growth and development, is involved in abscisic acid signaling. Plant Cell 18:3415-3428.

Tang, D., Christiansen, K. M., and Innes, R. W. 2005. Regulation of plant disease resistance, stress responses, cell death, and ethylene signaling in Arabidopsis by the EDR1 protein kinase. Plant Physiol. 138:1018-1026.

Ton, J., and Mauch-Mani, B. 2004. Beta-amino-butyric acid-induced resistance against necrotrophic pathogens is based on ABA-dependent priming for callose. Plant J. 38:119-130.

Ton, J., Jakab, G., Toquin, V., Flors, V., Iavicoli, A., Maeder, M. N., Metraux, J. P., and Mauch-Mani, B. 2005. Dissecting the betaaminobutyric acid-induced priming phenomenon in Arabidopsis. Plant Cell 17:987-999.

Tsuda, K., Sato, M., Glazebrook, J., Cohen, J. D., and Katagiri, F. 2008. Interplay between MAMP-triggered and SA-mediated defense responses. Plant J. 53:763-775.

van Hulten, M., Pelser, M., van Loon, L. C., Pieterse, C. M., and Ton, J. 2006. Costs and benefits of priming for defense in Arabidopsis. Proc. Natl. Acad. Sci. U.S.A. 103:5602-5607.

Verma, D. P., and Hong, Z. 2001. Plant callose synthase complexes. Plant Mol Biol 47:693-701.

Vernooij, B., Uknes, S., Ward, E., and Ryals, J. 1994. Salicylic acid as a signal molecule in plant-pathogen interactions. Curr. Opin. Cell Biol. 6:275-279.

Vogel, J., and Somerville, S. 2000. Isolation and characterization of powdery mildew-resistant Arabidopsis mutants. Proc. Natl. Acad. Sci. U.S.A. 97:1897-1902.

Wawrzynska, A., Christiansen, K. M., Lan, Y., Rodibaugh, N. L., and Innes, R. W. 2008. Powdery mildew resistance conferred by loss of the ENHANCED DISEASE RESISTANCE1 protein kinase is suppressed by a missense mutation in KEEP ON GOING, a regulator of abscisic acid signaling. Plant Physiol. 148:1510-1522.

Wildermuth, M. C., Dewdney, J., Wu, G., and Ausubel, F. M. 2001. Isochorismate synthase is required to synthesize salicylic acid for plant defence. Nature 414:562-565.

\section{AUTHOR-RECOMMENDED INTERNET RESOURCE}

Monsanto Col-0 and Ler polymorphism database: www.arabidopsis.org/Cereon/index.jsp 